\title{
Histologic Characterization of Kratom Use-Associated Liver Injury
}

\author{
Michael Riverso $^{\mathrm{a}}$, Michael Chang ${ }^{\mathrm{b}}$, Consuelo Soldevila-Pico ${ }^{\mathrm{a}}$, \\ Jinping Lai ${ }^{\mathrm{b}}$, Xiuli Liu ${ }^{\mathrm{b}, \mathrm{c}}$
}

\begin{abstract}
Kratom is an herbal product derived from the leaves of Southeast Asian Mitragyna speciosa trees. It has traditionally been used by indigenous people to relieve fatigue and manage pain, diarrhea, or opioid withdrawal. The use of kratom has become more commonplace in the United States for similar purposes. Only rare reports of kratom liver toxicity exist in the literature but without histologic characterization. Herein, we report one case of kratom use-associated liver toxicity in a 38-year-old patient. The patient complained of dark colored urine and light colored stools after using kratom. He had unremarkable physical examination. Laboratory testing at presentation revealed elevated alanine aminotransferase (389 U/L), aspartate aminotransferase $(220 \mathrm{U} / \mathrm{L})$, total bilirubin $(5.1 \mathrm{mg} / \mathrm{dL})$, and alkaline phosphatase (304 U/L). There was no serology evidence of viral hepatitis A, B, and C. The acetaminophen level at presentation was below detectable limits. Ultrasound examination of the right upper quadrant revealed normal echogenicity and contour of the liver without bile ductal dilatation or disease of the gallbladder. The patient underwent liver biopsy 4 days after the initial presentation which revealed a pattern of acute cholestatic liver injury including zone 3 hepatocellular and canalicular cholestasis, focal hepatocyte dropout, mild portal inflammation, and bile duct injury. Kratom was stopped, the patient improved clinically and biochemically and was discharged 8 days after the initial presentation. To our best knowledge, this is the first case report detailing the histology of kratom use-associated liver injury.
\end{abstract}

Keywords: Liver toxicity; Kratom; Cholestasis

\section{Introduction}

Kratom is an herbal product derived from the leaves of South-

Manuscript submitted January 11, 2018, accepted February 2, 2018

a Department of Gastroenterology, Hepatology, and Nutrition, University of Florida, Gainesville, FL, USA

bDepartment of Pathology, Immunology, and Laboratory Medicine, Gainesville, FL, USA

${ }^{\mathrm{c} C}$ Corresponding Author: Xiuli Liu, Department of Pathology, Immunology, and Laboratory Medicine, Gainesville, P.O. Box 100275, Gainesville, FL 32610, USA. Email: xiuliliu@ufl.edu

doi: https://doi.org/10.14740/gr990e east Asian Mitragyna speciosa trees. It has traditionally been used by indigenous people to relieve fatigue and manage pain, including muscle aches, diarrhea, or opioid withdrawal [1]. The use of kratom has become more commonplace in the United States and other Western countries for similar purposes, mainly to counteract fatigue, muscle aches, and relieve opioid withdrawal symptoms [2]. Only rare reports of kratom toxicity exist in the literature $[3,4]$. However, thus far, histologic characterization of kratom use-associated liver toxicity has not been reported with illustration. This case report describes the histologic features in a liver biopsy from a patient taking kratom.

\section{Case Report}

A 38-year-old man with no significant past medical history presented to the emergency department (ED) complaining of dark colored urine and light colored stools. The patient claimed he had a fever and chills 5 days prior to this visit, which prompted a visit to the ED, where the patient was told he likely had a viral infection and was sent home with Tylenol. At the current presentation, the patient endorsed having taken five doses of Tylenol. The patient's symptoms initially improved, but then he noted changes in the color of his urine and stool. He claimed he did not take any other prescription medication, but he had been taking the herbal drug kratom for malaise/fatigue before his initial presentation and also while he was on the Tylenol. The patient returned to the hospital for evaluation. He noted the sudden onset of sternal pleuritic chest pain while sitting in the ED, as well as mild shortness of breath. The chest pain was non-radiating and did not increase on palpation. The patient acknowledged that he was very anxious after learning about the potential for liver injury in kratom users. He had a mild cough, but denied having phlegm, sore throat, or rhinorrhea. The patient also denied experiencing nausea, vomiting, fever, abdominal pain, and bleeding. The physical examination was unremarkable. The patient was alert and oriented to person, place, and time. No scleral icterus was present. The skin was unremarkable and without jaundice. The abdomen was soft with normal bowel sounds. No distention, mass, tenderness, rebound, or guarding was noted. A computed tomography $(\mathrm{CT})$ of the abdomen and pelvis from 3 years prior was unremarkable.

Ultrasound examination of the right upper quadrant revealed normal hepatic echogenicity and a normal contour 
Table 1. Laboratory Values at the Time of Presentation and After Cessation of Kratom

\begin{tabular}{lllllll}
\hline & ALT (U/L) & AST (U/L) & Bilirubin (mg/dL) & ALP (U/L) & GGT (U/L) & Comment \\
\hline July16, 2016 & 389 & 220 & 5.1 & 304 & Not done & Kratom stopped \\
July 18, 2016 & 360 & 166 & 5.6 & 266 & 394 & 241 \\
July 24, 2016 & 410 & 142 & 1.6 & 266 & $35-129$ & $5-61$ \\
\hline Normal values & $0-41$ & $0-37$ & $0.0-1.0$ & & \\
\hline
\end{tabular}

without hepatic lesions. No intrahepatic or extrahepatic biliary ductal dilatation was present. The main portal vein was patent, with normal hepatopedal flow. The gallbladder was nondistended, without cholelithiasis or wall thickening. No sonographic Murphy sign was noted. The common bile duct measured $4 \mathrm{~mm}$ in diameter. The observed portions of the pancreas were normal.

Laboratory testing on July 16, 2016 revealed the following values: alanine aminotransferase (ALT) 389 (0 - $41 \mathrm{U} / \mathrm{L})$; aspartate aminotransferase (AST) 220 (0 - $37 \mathrm{U} / \mathrm{L})$; total bilirubin $5.1(0.0-1.0 \mathrm{mg} / \mathrm{dL})$; direct bilirubin $4.0(0.0-0.2 \mathrm{mg} /$ dL); alkaline phosphatase (ALP) 304 (35 - $129 \mathrm{U} / \mathrm{L})$; total protein 7.8 (6.4 - $8.3 \mathrm{~g} / \mathrm{dL})$; albumin $4.2(3.5-5.0 \mathrm{~g} / \mathrm{dL})(\mathrm{Ta}-$ ble 1). Gamma-glutamyl transferase (GGT) on July 18, 2016 was 394 (5 - 61 U/L) (Table 1). The acetaminophen level on July 16, 2016 was below detectable limits $(10-25 \mu \mathrm{g} / \mathrm{mL})$. The serum alpha-1 antitrypsin level on July 22, 2016 was 188 (90 - $200 \mathrm{mg} / \mathrm{dL})$ and he had a normal phenotype (M1M2; July 22, 2016). Hepatitis A total antibody was positive (July 21, 2016), and hepatitis B surface antibody was positive (July 19, 2016). Hepatitis B DNA and hepatitis C RNA were not detected (July 22, 2016). His serum IgG was $710 \mathrm{mg} / \mathrm{dL}$ (716 - $1,554 \mathrm{mg} / \mathrm{dL})$. His serum $\operatorname{IgA}$ and $\operatorname{IgM}$ were within normal range.

The patient underwent a percutaneous liver biopsy on July 20, 2016 due to sustained elevation of ALT, AST, and total bilirubin (360 U/L, $166 \mathrm{U} / \mathrm{L}, 5.6 \mathrm{mg} / \mathrm{dL}$ on July 19, 2016). The biopsy specimen measured $1.5 \mathrm{~cm}$ in length and contained 22 completely sampled portal tracts and two incomplete portal tracts. The biopsy showed normal lobular architecture (Fig. 1a). Regenerative nodules were absent. Portal tracts contained a mild inflammatory infiltrate with a conspicuous population of eosinophils, as well as some neutrophils, lymphocytes, and rare plasma cells (Fig. 1b). Portal bile ducts had mild injury with rare apoptotic bodies (Fig. 1c) and lymphocytic infiltration (Fig. 1d). Mild ductular proliferation was present. Mild focal endotheliitis was noted in one portal vein; however, the remaining portal veins were not involved by inflammation. No significant interface activity or portal edema was noted. The hepatic arteries were largely unremarkable. Focal steatosis (about $3-5 \%$ of biopsy volume) was noted (Fig. 1e). There was focal hepatocyte dropout both in a periportal and intralobular distribution, along with clusters of ceroid pigment-laden macrophages. The lobules had some degree of small cell change and rare mitotic figures. There was mild zone 3 hepatocellular and canalicular cholestasis (Fig. 1f). No Mallory hyaline, ballooning degeneration, or central zone necrosis was identified. Central veins are histologically unremarkable. A Prussian blue iron stain was negative for iron accumulation. PAS and PAS/D stains were negative for diagnostic cytoplasmic inclusions. A Masson trichrome stain showed focal portal fibrous expansion. The biopsy was interpreted as consistent with acute cholestatic liver injury associated with kratom use. The patient was initially treated with intravenous fluid, and kratom was stopped on July 16, 2016. The patient appeared stable overall and was discharged 8 days (July 24, 2016) after admission, at which time liver function tests improved (ALT $410 \mathrm{U} / \mathrm{L}$, AST 142 $\mathrm{U} / \mathrm{L}$, total bilirubin $1.6 \mathrm{mg} / \mathrm{dL}$, ALP $266 \mathrm{U} / \mathrm{L}$, GGT $241 \mathrm{U} / \mathrm{L}$ ) (Table 1).

\section{Discussion}

Kratom, an herbal medicine used with increasing frequency, has been reported to cause liver injury $[3,4]$. One report with liver biopsy briefly mentioned a so-called pure cholestasis without hepatocellular damage on histology [4]. According to the report, the bile was noted to precipitate in hepatocytes and in fat vacuoles of single steatotic hepatocytes; the sinusoids were slightly distended and hyperemic, with evidence of modest inflammation indicating a diagnosis of canalicular cholestasis [4]. However, no histologic pictures were presented in the report to illustrate these features.

The overall clinical presentation and clinical course in our current case support the diagnosis of liver injury associated with kratom use. Although the patient used acetaminophen for 5 days prior to his presentation, the dose was low, and his acetaminophen level at admission was undetectable. In addition, the lack of significant hepatocyte injury on biopsy makes acetaminophen liver toxicity in this case very unlikely. No biliary obstruction was noted on ultrasound examination. Serologic testing for viral hepatitis (HAV, $\mathrm{HBV}$, and $\mathrm{HCV}$ ) was negative.

This case report illustrates the histologic features of kratom-induced liver injury, which are characterized by mild portal mixed inflammation and lymphocyte-mediated bile duct injury. The lymphocyte-mediated injury also involves ductules. Although no follow-up biopsy was performed, the improvement of liver function tests, particularly total bilirubin (from 5.1 to $1.6 \mathrm{mg} / \mathrm{dL}$ ) (Table 1), 8 days after cessation of kratom suggests that kratom-associated liver injury is reversible with cessation of the drug.

\section{Disclosure}

None. 


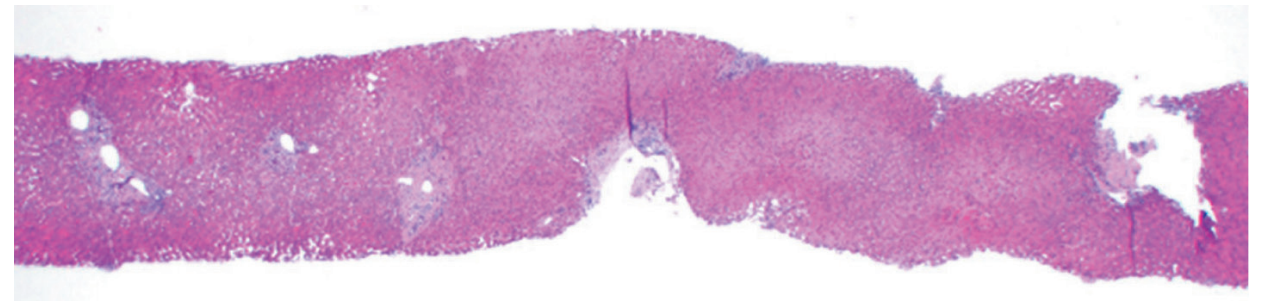

a
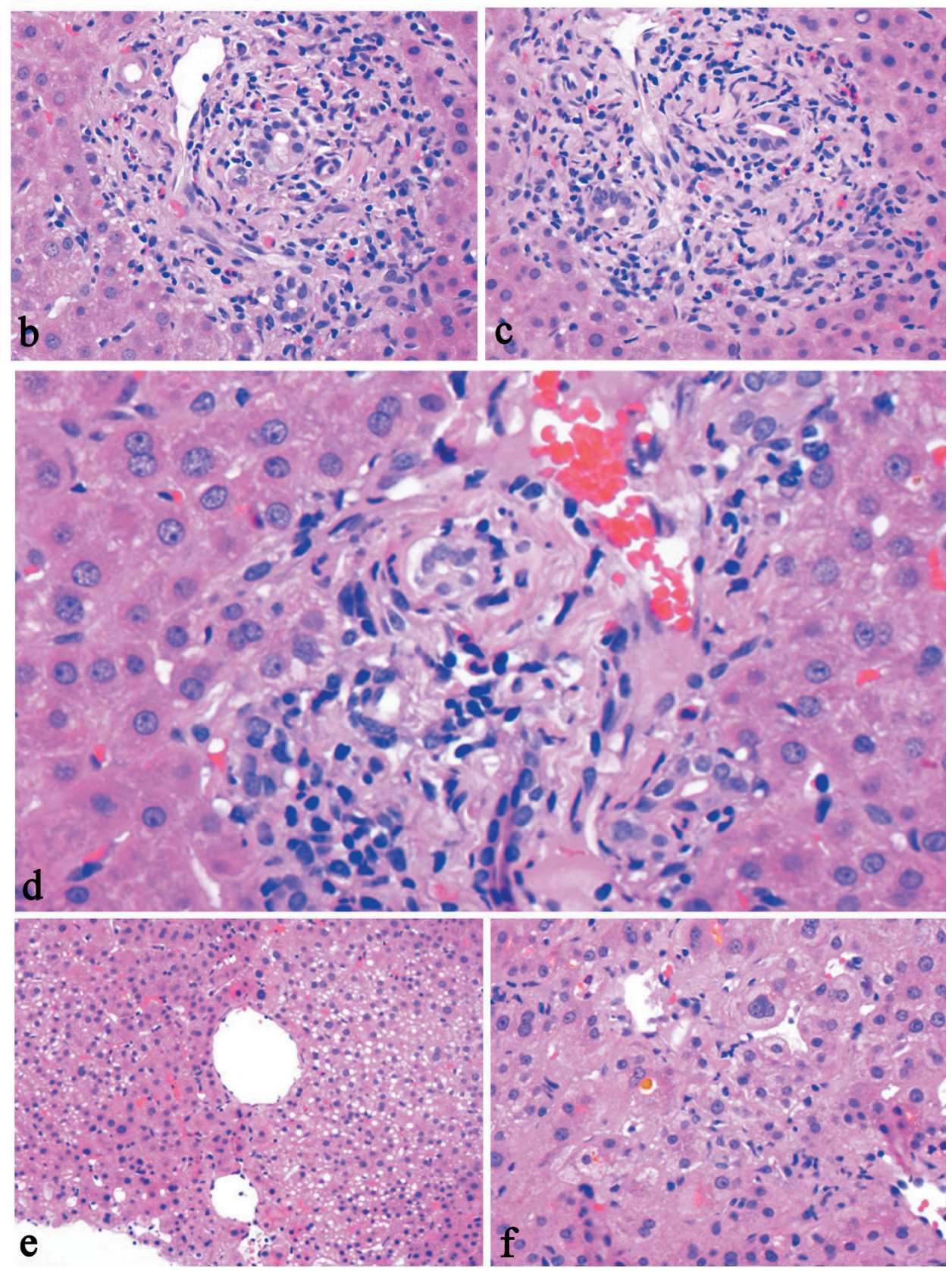

Figure 1. Histologic features of kratom-associated liver injury. The liver biopsy shows preserved normal lobular architecture (a, H\&E stain, 20x), mixed portal inflammation composed of lymphocytes, eosinophils, rare plasma cells, and rare neutrophils (b, H\&E stain, 200×), bile duct epithelial apoptosis (c, H\&E stain, 200x), lymphocytic inflammation involves bile duct and ductules (d, H\&E stain, 400×), focal steatosis (e, H\&E stain, 100x), and zone 3 intrahepatocellular and canalicular cholestasis (f, H\&E stain, 200x). 


\section{Grant Support}

None.

\section{References}

1. Prozialeck WC, Jivan JK, Andurkar SV. Pharmacology of kratom: an emerging botanical agent with stimulant, analgesic and opioid-like effects. J Am Osteopath Assoc.
2012;112(12):792-799.

2. Forrester MB. Kratom exposures reported to Texas poison centers. J Addict Dis. 2013;32(4):396-400.

3. Dorman C, Wong M, Khan A. Cholestatic hepatitis from prolonged kratom use: a case report. Hepatology. 2015;61(3):1086-1087.

4. Kapp FG, Maurer HH, Auwarter V, Winkelmann M, Hermanns-Clausen M. Intrahepatic cholestasis following abuse of powdered kratom (Mitragyna speciosa). J Med Toxicol. 2011;7(3):227-231. 